\title{
Modélisation de l'évolution spatiotemporelle du phosphore minéral dans une baie lagunaire hypereutrophe tropicale : la baie lagunaire de Tiagba (Côte d'Ivoire) Spatio-temporal evolution of mineral phosphorus modelling in tropical hypereutrophic lagoon bay: Tiagba Lagoon Bay (Côte d'Ivoire)
}

\author{
Marcel Konan Yao, Djedro Clément Akmel, Kouamé Lazare Akpetou, Albert \\ Trokourey, Kouassi Benjamin Yao et Nogbou Emmanuel Assidjo
}

Volume 30, numéro 3, 2017

Reçu le 17 novembre 2015, accepté le 19 avril 2017

URI : https://id.erudit.org/iderudit/1044250ar

DOI : https://doi.org/10.7202/1044250ar

Aller au sommaire du numéro

Éditeur(s)

Université du Québec - INRS-Eau, Terre et Environnement (INRS-ETE)

ISSN

1718-8598 (numérique)

Découvrir la revue

Citer cet article

Yao, M. K., Akmel, D. C., Akpetou, K. L., Trokourey, A., Yao, K. B. \& Assidjo, N. E. (2017). Modélisation de l'évolution spatiotemporelle du phosphore minéral dans une baie lagunaire hypereutrophe tropicale : la baie lagunaire de Tiagba (Côte d'Ivoire). Revue des sciences de l'eau / Journal of Water Science, 30(3), 247-258. https://doi.org/10.7202/1044250ar
Résumé de l'article

Ce travail décrit une nouvelle approche de la prédiction de l'évolution spatio-temporelle du phosphore minéral dans les eaux de surface, particulièrement dans la baie lagunaire de Tiagba. L'originalité de cette étude réside dans l'utilisation des réseaux de neurones artificiels, précisément du perceptron multicouche, comme outil de modélisation. Deux approches de l'évolution spatio-temporelle de ce nutriment dans cette baie ont été étudiées : sa modélisation statique et sa modélisation dynamique. Ainsi, il a été utilisé deux bases de 3966 et 4627 données respectivement pour sa modélisation statique et sa modélisation dynamique. L'algorithme de Levenberg-Marquardt a été utilisé pour la détermination des poids de connexions lors du développement du perceptron multicouche. Il ressort, des résultats obtenus, que les modèles 5-14-1 et 6-14-2 permettent de prédire à 70,30 \% et à environ $70 \%$ respectivement les évolutions statique et dynamique du phosphore minéral dans cette baie lagunaire. Ces modèles, jugés satisfaisant peuvent servir de socle pour d'éventuelles études visant à la réhabilitation et la gestion de cet écosystème aquatique dans le cadre de son développement durable. 


\section{MODÉLISATION DE L'ÉVOLUTION SPATIOTEMPORELLE} DU PHOSPHORE MINÉRAL DANS UNE BAIE LAGUNAIRE HYPEREUTROPHE TROPICALE : LA BAIE LAGUNAIRE DE TIAGBA (CÔTE D'IVOIRE)

Spatio-temporal evolution of mineral phosphorus modelling in tropical hypereutrophic lagoon bay: Tiagba Lagoon Bay (Côte d'Ivoire)

Marcel Konan YAO ${ }^{\prime *}$, Djedro Clément AKMEL², Kouamé Lazare AKPETOU ${ }^{3}$, Albert TROKOUREY', Kouassi Benjamin YAO², Nogbou EMmanuel ASSIDJO²

${ }^{1}$ Université Félix Houphouët-Boigny, Laboratoire de Chimie-Physique, 22 BP 582, Abidjan 22, Côte d'Ivoire

${ }^{2}$ Institut national polytechnique Félix Houphouët-Boigny, Laboratoire des procédés Industriels, de Synthèse, de l'Environnement et des Énergies nouvelles, BP 1093, Yamoussoukro, Côte d'Ivoire

${ }^{3}$ Université Jean Lorougnon Guédé, UP de Chimie-Physique-Mathématiques, 12 BP V 25, Daloa 12, Côte d'Ivoire

Reçu le 17 novembre 2015, accepté le 19 avril 2017

\section{RÉSUMÉ}

Ce travail décrit une nouvelle approche de la prédiction de l'évolution spatio-temporelle du phosphore minéral dans les eaux de surface, particulièrement dans la baie lagunaire de Tiagba. L'originalité de cette étude réside dans l'utilisation des réseaux de neurones artificiels, précisément du perceptron multicouche, comme outil de modélisation. Deux approches de l'évolution spatio-temporelle de ce nutriment dans cette baie ont été étudiées : sa modélisation statique et sa modélisation dynamique. Ainsi, il a été utilisé deux bases de 3966 et 4627 données respectivement pour sa modélisation statique et sa modélisation dynamique. L'algorithme de Levenberg-Marquardt a été utilisé pour la détermination des poids de connexions lors du développement du perceptron multicouche. Il ressort, des résultats obtenus, que les modèles 5-14-1 et 6-14-2 permettent de prédire à 70,30 \% et à environ
$70 \%$ respectivement les évolutions statique et dynamique du phosphore minéral dans cette baie lagunaire. Ces modèles, jugés satisfaisant peuvent servir de socle pour d'éventuelles études visant à la réhabilitation et la gestion de cet écosystème aquatique dans le cadre de son développement durable.

Mots-clés : Phosphore, réseau de neurones artificiels, baie lagunaire de Tiagba, système Ébrié, Côte d'Ivoire.

\begin{abstract}
This work describes a new approach to prediction of spatio-temporal evolution of mineral phosphorus in water bodies, particularly in Tiagba Lagoon Bay. Originality of this
\end{abstract}


study lie on the use of artificial neural networks, principally multilayer perceptron, as modelling tool. Two approaches of spatio-temporal evolution of this nutrient were done: static evolution and dynamic evolutions. Data bases, formed by 3966 and 4627 data, served for static and dynamic modelling of this nutrient respectively. Weights of network connection are determined using Levenberg-Marquardt algorithm during execution of Multilayer Perceptron. Results obtained show that models 5-14-1 and 6-14-2 can predict to $70.30 \%$ and approximately $70 \%$ respectively for static and dynamic evolution of mineral phosphorus in this bay. These models, judged satisfactory, could be used for other studies led to rehabilitation and protection of this aquatic ecosystem for its long development.

Key Words: Phosphorus, artificial neural networks, multilayer perceptron, Tiagba Lagoon Bay, Ebrié system, Côte d'Ivoire

\section{INTRODUCTION}

Le phosphore est un élément essentiel à l'écosystème. Dans les écosystèmes aquatiques, le phosphore est présent sous forme dissoute, particulaire et sédimentaire (LIU et al., 2016a; ZUO et al., 2016). Quelle que soit la forme considérée, il y est présent sous forme de phosphore minéral et de phosphore organique. Le phosphore organique, correspondant à des molécules de phosphates associées à des molécules à base carbonée, représente la forme dominante dans les eaux de surface. Quant au phosphore minéral, il correspond aux polyphosphates minéraux d'une part, et les orthophosphates $\left(\mathrm{H}_{\mathrm{n}} \mathrm{PO}_{4}^{3-\mathrm{n}}\right)$ représentant sa forme la plus importante et la seule soluble à être utilisé par les organismes aquatiques (CAO et al., 2016; LIU et al., 2016b) d'autre part.

Si le phosphore constitue l'un des éléments nutritifs majeurs pour les écosystèmes aquatiques, il n'en demeure pas moins que sa présence excessive dans ces entités est l'une des causes principales de l'eutrophisation. Dans ce fléau écologique, il est incriminé en tant que cofacteur limitant avec l'azote (GOODY et al., 2016; KASPERSEN et al., 2016; ROLLAND et al., 2015; ULRICH et al., 2016). Il en est le facteur de maîtrise du fait de son cycle biogéochimique (ABEL et al., 2010; YAO, 2011). En effet, l'eutrophisation, se traduisant par une prolifération anarchique de végétaux aquatiques de tous genres, est responsable des dégradations abiotiques et biotiques des eaux de surface (CABRITA et al., 2015; PINEDO et al., 2015; PIZARRO et al., 2016; SUTELA et al., 2016). Ainsi, le phosphore a toujours suscité d'importants intérêts d'étude dans les écosystèmes aquatiques.
La dynamique du phosphore dans les eaux de surface, diverse et typique à chacune d'elles, illustre la complexité de son cycle biogéochimique. Si pour les eaux de surface à petits bassins versants (lac, réservoir, étang, etc.), une approche du cycle biogéochimique de ce nutriment est relativement satisfaisante (MELLANDER et al., 2016; SUNOHARA et al., 2016; WANG et LIANG, 2016; YAO et al., 2016a), ce n'est pas le cas pour celles à grands bassins versants (fleuves, lagunes, océans, etc.). Pour ces entités, il est fait recours à la modélisation tant pour apprécier les facteurs (exogènes et endogènes) intervenant dans son cycle biogéochimique que pour extrapoler son évolution en leur sein et sur leur bassin versant. La modélisation du phosphore dans les eaux de surface connait un essor remarquable depuis 1990 eu égard à la croissance vertigineuse des modèles consacrés au phosphore. Létude réalisée par ROBSON (2014) a montré que la quasitotalité des modèles consacrés à ce sujet sont des modèles de connaissances. Ainsi, les modèles de type «boite noire " semblent -ils être lésés en dépit de leur performance reconnue dans tous les domaines. Parmi ceux-ci figurent les réseaux de neurones artificiels (RNA). Ce véritable outil statistique connait depuis toujours un grand succès dans la modélisation des phénomènes et des procédés. Cette situation s'explique par sa parcimonie et sa rapidité qui permet de les généraliser (MANDAL et al., 2015; XU et al., 2015). Plusieurs variantes de RNA existent, dont le plus connu est le perceptron multicouche (PMC). La capacité de ce type de RNA à modéliser les problèmes aquatiques n'est plus à démontrer, particulièrement l'eutrophisation (CHANG et al., 2015; HUANG et GAO, 2017; HUO et al., 2013; MA et al., 2014; MULLER et MULLER, 2015; SHOULIANG et al., 2013). Le PMC se caractérise par sa typologie et son fonctionnement. En effet, il possède une seule couche d'entrée, au moins une couche cachée et une couche de sortie. Il est généralement utilisé avec une seule couche cachée du fait que certains travaux ont montré que dans cette configuration il permet d'approximer n'importe quelles fonctions non linéaires et complexes (ZHANG, 2003). Chaque neurone artificiel est un mini processeur qui calcule la somme des données reçues à son entrée et fournit le résultat de calcul à sa sortie. Il n'y a pas de connexions entre les neurones artificiels d'une même couche. Chaque connexion est pondérée par un " poids ". Il n'est pas défini de fonction sur la couche d'entrée. Cependant, sur la(les) couche(s) cachée(s) il est défini une fonction d'activation, et sur la couche de sortie, la fonction simple de linéarité. Le réseau est synchronisé et non rétroactif (AICHOURI et al., 2015).

À l'instar de tout le système Ébrié, la baie de Tiagba est sujette à de fortes pressions anthropiques. Cette situation entraine sa dégradation, traduite par son état d'hypereutrophisation (YAO, 2011). Une autre illustration est apportée par le taux de croissance du phosphore $(+0,144$ pour le phosphore total et $+0,636$ pour le phosphore minéral) dans cette baie lagunaire sur ce dernier quart de siècle, noté en comparant ses teneurs moyennes obtenues par CARMOUZE et CAUMETTE 
(1985) sur la période de 1976 à 1979 à celles de YAO (2011) sur la période de 2006 à 2009. À cet effet, il importe de mener des actions visant sa réhabilitation et sa protection dans le cadre de son développement durable. Ce fait passe d'abord par la connaissance d'un certain nombre de processus liéà sa pollution. C'est dans cette optique que l'évolution spatiotemporelle du phosphore minéral dans les eaux de cette baie lagunaire a été modélisée par le PMC.

\section{MATÉRIEL ET MÉTHODES}

\subsection{Présentation de la zone d'étude}

La baie lagunaire de Tiagba est localisée précisément entre $4^{\circ} 40^{\prime}$ et $4^{\circ} 45^{\prime}$ de longitude ouest à la latitude nord de $5^{\circ} 20^{\prime}$ et à $73 \mathrm{~m}$ au-dessus du niveau de la mer. Ses différentes caractéristiques morphologiques et hydrologiques sont données à la figure 1. Située à $80 \mathrm{~km}$ du canal de Vridi (unique entrée des eaux marines dans le système Ébrié), cette baie est soumise à une influence amoindrie de l'océan Atlantique. Son taux de renouvellement, faible (0,24 par an), est essentiellement dû aux apports météorites. Ses apports, relativement faibles, sont du fait de la rivière Ira par le biais de la baie de Cosrou via la passe de «Cosrou » et du fleuve Bandama par le truchement du canal d'Assagny et de la passe d' " eaux libres du chenal central » d'une part, et des eaux météorites de ruissellement du bassin versant d'autre part.

\subsection{Choix des paramètres pertinents}

En se référant aux travaux de YAO (2011), les paramètres interagissant avec la dynamique du phosphore dans les eaux de la baie de Tiagba sont à priori : la température, le $\mathrm{pH}$, le potentiel d'oxydoréduction, la transparence, les matières en suspension (MES), l'oxygène dissous, la chlorophylle $a$, la pluviométrie dans la zone d'étude, le débit du fleuve Bandama et le débit de la rivière Ira. Le choix définitif des paramètres jugés pertinents dans la dynamique du phosphore minéral dans cette baie lagunaire a été fait à partir de l'analyse en composantes principales (ACP). L'ACP a été appliquée sur une base de 6610 données composées de 661 échantillons (observations). Dans cette étude, l'ACP a été réalisée à partir du logiciel Statistica version 7.1.

\subsection{Développement du PMC dans cette étude}

Le développement du PMC dans cette étude a été réalisé à partir du logiciel Matlab R2015a conjointement avec le module Statistics and Machine Learning Toolbox.

\subsubsection{Scénarios étudiés}

Deux scénarios ont été abordés dans le cadre de cette étude :

- Scénario $1:$ modélisation statique du phosphore minéral. Le seul paramètre de sortie est la teneur en phosphore minéral $(Y 1)$, tandis que les teneurs des paramètres jugés pertinents constituent les paramètres d'entrée $\left(X_{i}\right)$;

- Scénario 2 : modélisation dynamique du phosphore minéral. Deux paramètres de sortie sont utilisés dans ce cas, à savoir les teneurs en phosphore minéral aux temps $t$ (mois en cours $Y[t]$ ) et à la date $t+1$ (mois suivant $Y[t+1]$ ). Aux teneurs des paramètres pertinents, il est ajouté le paramètre temps (mois) pour constituer les paramètres d'entrée $\left(X_{i}\right)$.

Au cours de cette étude, les teneurs des différents paramètres utilisées sont celles fournies par YAO (2011) sur la période 2007-2009. Le paramètre temps (mois) a été codé entre 0 et 22.

\subsubsection{Conditions du déroulement du PMC}

D’une manière générale, les bases de données doivent subir un prétraitement afin d'être adaptées aux entrées et sorties des modèles mathématiques stochastiques, tel que le PMC. Un prétraitement courant consiste à effectuer une normalisation appropriée qui tient compte de l'amplitude des valeurs acceptées par les modèles (BASHEER et HAJMEER, 2000; MANSSOURI et al., 2015). Cette base de données est constituée de différentes variations des variables indépendantes (entrées du modèle) et dépendantes (sortie du modèle). Dans cette étude, les différents paramètres ont été normalisés suivant l'équation 1 et codés dans un intervalle compris entre 0 et $n$ ( $n$ appartenant aux entiers naturels) par le logiciel Matlab R2015a conjointement avec le module Statistics and Machine Learning Toolbox.

$$
x n_{i}=\frac{2\left(x_{i}-x_{\min }\right)}{\left(x_{\max }-x_{\min }\right)}-1
$$

où $x n_{i}$ est la donnée normalisée, comprise entre -1 et $+1, x_{i}$ est la donnée à sa valeur initiale, $x_{\max }$ est la valeur maximale de l'ensemble de données et $x_{\min }$ est la valeur minimale de l'ensemble de données.

Pour l'obtention de faibles erreurs de prédiction, les poids $\mathrm{du}$ réseau sont initialisés avant leur modification au cours du processus d'apprentissage. L'architecture du réseau a été optimisée en faisant varier le nombre de couches cachées de 1 à 15 en tenant compte des coefficients de corrélation $(R)$ et de la moyenne carré des erreurs (MCE ). Pour chaque valeur de la couche cachée, les simulations ont été effectuées 1500 fois et le meilleur résultat de l'architecture du réseau correspondant a été enregistré. Ce meilleur résultat correspond à celui présentant simultanément les plus fortes valeurs de $R$ lors des 


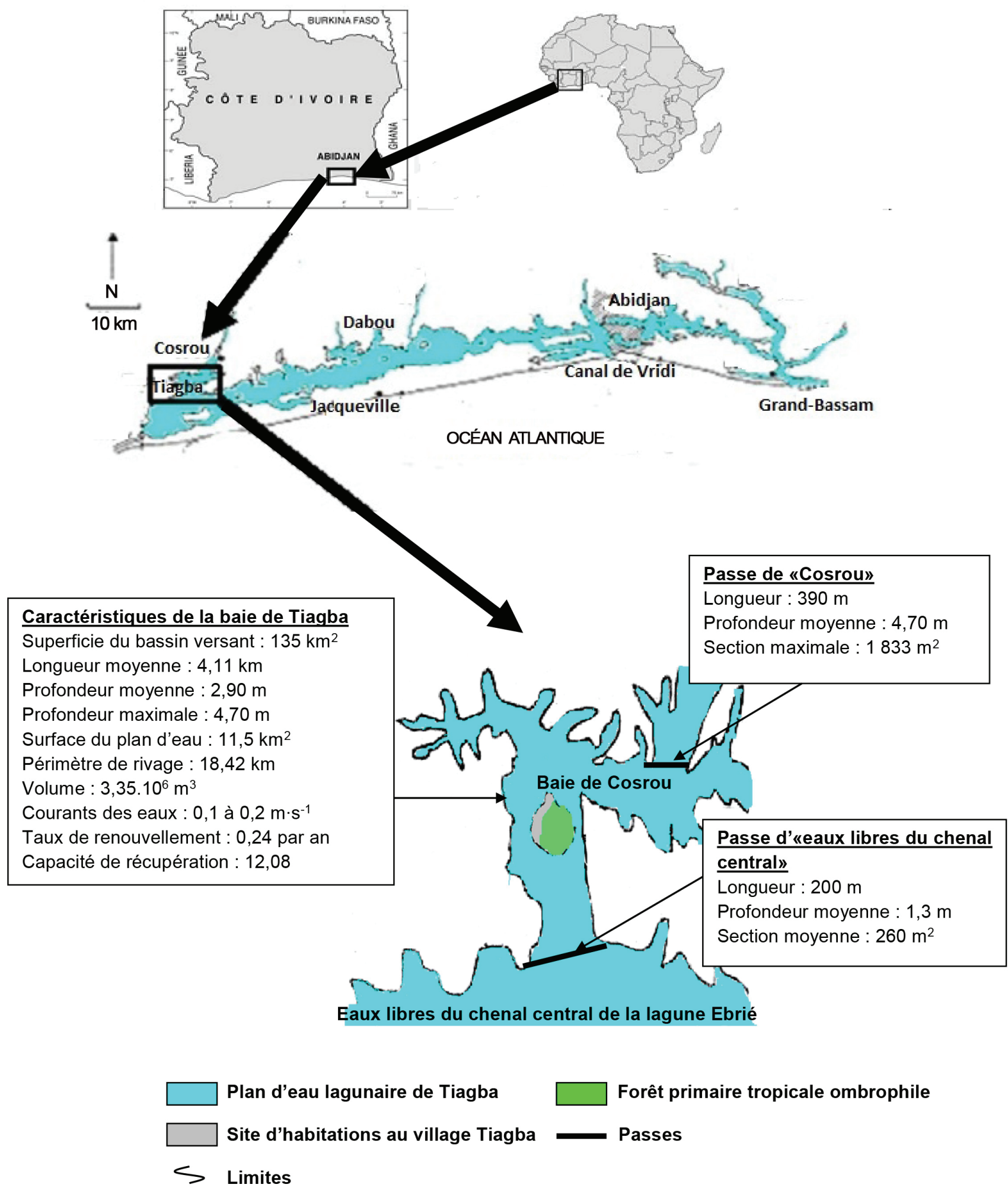

Figure 1. Caractéristiques morphologiques et hydrologiques de la baie lagunaire de Tiagba. Morphologic and hydrologic characteristic of Tiagba Lagoon Bay. 
phases d'apprentissage $\left(R_{a p p}\right)$ et de validation $\left(R_{v a l}\right)$, mais aussi les plus faibles valeurs de MCE dans ces deux phases. Durant toutes les simulations, l'algorithme de Levenberg-Marquardt (LEVENBERG, 1944; MARQUARDT, 1963) a été utilisé pour accélérer la phase d'apprentissage. Le taux d'apprentissage est fixé au départ à 0,2 et progressivement réduit à 0,01 à des pas de 0,05 . À cet effet, $50 \%$ et $25 \%$ de la base de données ont été respectivement utilisés pour l'exécution des phases d'apprentissage et de validation. La fonction de transfert utilisée entre la couche d'entrée et la couche de sortie est la tangente hyperbolique (Tanh). Ainsi, chaque neurone caché produit-il une sortie $Y_{i}$ selon l'équation 2 :

$$
Y_{i}=\operatorname{Tanh}\left(\sum x_{i} w_{i}+\beta i\right)
$$

où $w_{i}$ représente les poids, $x_{i}$ les entrées et $\beta_{i}$ le biais associé à la sortie $Y_{i}$

La fonction linéaire simple $(y=x)$ est utilisée comme fonction de transfert entre la couche cachée et la couche de sortie. De ce fait, chaque neurone de sortie produit une sortie $Y_{j}$ suivant l'équation 3 :

$$
Y_{j}=\sum y_{i} w_{j}+\beta_{j}
$$

où $w_{j}$ représente les poids, $y_{i}$ les neurones cachés et $\beta_{j}$ le biais associé à la sortie $Y_{j}$.

\subsubsection{Choix du modèle et conditions de sa validation finale}

\subsubsection{Choix du modèle}

Le meilleur modèle traduisant au mieux le phénomène étudié correspond à celui présentant simultanément les valeurs les plus élevées de $R_{a p p}$ et de $R_{v a l}$ d'une part, et la plus faible valeur de la MCE lors de la phase de validation $\left(\mathrm{MCE}_{\text {val }}\right)$ d'autre part. Cependant, si les valeurs les plus élevées de $R_{a p p}$ et $R_{v a p}$ et la plus faible valeur de $\mathrm{MCE}_{\text {val }}$ sont distinctement obtenues pour des modèles différents, le meilleur compromis est celui qui présente la plus grande valeur de $R$ (Équation 4), mais aussi une $\mathrm{MCE}_{\text {val }}$ parmi les plus faibles pour les modèles ayant une sortie. Pour ceux ayant deux sorties, le meilleur modèle est celui présentant la plus grande valeur de $R^{\prime}$ (Équation 5) et ayant une $\mathrm{MCE}_{\text {val }}$ parmi les plus faibles (YAO et al., 2016a). $R$ et $R$ ' sont donnés par :

$$
\begin{aligned}
R & =\frac{R_{a p p}+R_{v a l}}{2} \\
R^{\prime} & =\frac{\sum R}{2}
\end{aligned}
$$

où $R$ est la moyenne arithmétique de $R_{a p p}$ et $R_{v a l}$ et $R^{\prime}$ est la moyenne arithmétique de $R$ pour deux sorties du PMC.
2.2.2.2 Validation finale du modèle

La validation finale du modèle consiste à juger sa capacité de prédiction de la dynamique du phosphore dans la baie lagunaire de Tiagba. Cela en utilisant les poids et les biais calculés durant l'apprentissage pour les appliquer à une autre base de données. Cette phase du PMC s'appelle le "test ". Vingt-cinq pourcent (25\%) de la base de données, normés suivant l'équation 1, ont été utilisés à cet effet.

Le modèle choisi est validé finalement si les conditions suivantes sont simultanément satisfaites :

- $\quad R_{\text {val }}^{2}>0,5$ (YAO, 2011; YAO et al., 2016a), en d'autres termes si le modèle traduit à plus de $50 \%$ le phénomène étudié lors de la phase de validation;

- la MCE et la racine des moyennes des carrés des erreurs (RMCE ) lors de la phase de test doivent être relativement très faibles;

- l'erreur moyenne de prévision $\left(e_{\text {moo }}\right)$ entre les valeurs de phosphore minéral obtenues expérimentalement $\left(Y_{o b s}\right)$ et celles prédites $\left(Y_{p}\right)$ par le modèle lors du test, doit être relativement très faible. Cette erreur est calculée suivant l'équation 6 :

$$
e_{m o y}=\frac{\sum_{1}^{N}\left(Y_{p r}-Y_{o b s}\right)}{N}
$$

où $N$ est le nombre d'échantillons dans la base de données $\mathrm{du}$ « test ».

- l'erreur moyenne relative de déviation $(\Delta[\%])$ entre les valeurs de phosphore minéral obtenues expérimentalement $\left(Y_{o b s}\right)$ et celles prédites par le modèle $\left(Y_{p r}\right)$ lors du test doit être inférieur à $10 \%$ tel recommandé pour les modèles statistiques (NOUMI, 2004; YAO, 2011). Elle s'obtient selon l'équation 7 :

$$
\Delta(\%)=\frac{100}{N} \times \sum_{1}^{N}\left(\frac{Y_{p r}-Y_{o b s}}{Y_{o b s}}\right)
$$

où $N$ est le nombre d'échantillons dans la base de données du « test».

\section{RÉSULTATS ET DISCUSSION}

\subsection{Résultats}

\subsubsection{Choix des paramètres pertinents}

Dans cette étude, cinq paramètres ont été jugés pertinents dans la dynamique du phosphore à l'issue de l'application de l'ACP aux dix paramètres préalablement choisis à la section 2.2. 
Il s'agit de la transparence, des MES, de la chlorophylle $a$, de la pluviométrie dans la zone d'étude et du débit du Bandama.

Le tableau 1 présente les valeurs propres et les statistiques associées de l'ACP obtenu à partir de ces cinq paramètres et du phosphore minéral. Les deux premiers facteurs à eux seuls représentent $56,22 \%$ de la variance totale, dont 34,14\% pour $\mathrm{F} 1$ et $17,38 \%$ pour F2. Le facteur F1 est très important par rapport aux autres. Quant au tableau 2, relatif à la matrice de corrélation de Bravais-Pearson, il montre une très forte corrélation entre le phosphore minéral et la transparence $(r=$ $1,00)$. Cette corrélation est très significative et témoigne de l'affinité entre ces deux éléments. Par contre, les corrélations du phosphore minéral avec les MES $(r=0,14)$ et la chlorophylle $a$ $(r=0,08)$ sont très faibles. Il en est de même pour le débit du Bandama avec les MES $(r=-0,11)$ et la pluviométrie $(r=0,13)$, de la chlorophylle $a$ avec les MES $(r=0,10)$ et le débit du Bandama $(r=0,29)$, des MES avec la transparence $(r=0,13)$.

La projection de ces paramètres dans le plan factoriel F1-F2, met en évidence deux regroupements des paramètres étudiés dans les échantillons d'eau (Figure 2) :

- $G_{1}$, contenant le phosphore minéral, la transparence et les MES;
- $G_{2}$, regroupant la chlorophylle $a$, la pluviométrie dans la zone d'étude et le débit mensuel du Bandama.

$G_{1}$ traduit partiellement les processus intervenant dans la minéralisation de la matière organique, tandis que $G_{2}$ exprime partiellement les facteurs influençant les activités photosynthétiques. Ces deux entités sont complémentaires. Cependant, leur affinité dans ce cas d'étude tend à être masquée par l'état hypereutrophe de la baie lagunaire de Tiagba. Ainsi, le facteur F1 semble-t-il rendre compte des conditions d'acquisition du chimisme du phosphore minéral. Ce facteur est déterminé par le phosphore minéral et la transparence. En effet, la luminosité est d'une grande importance dans la dégradation et la minéralisation de la matière organique (COBLE et al., 2015; FOTIOU et al., 2015; SONG et al., 2016), donc à la production du phosphore minéral.

Ces paramètres permettent de former des bases de 3966 données pour la modélisation statique et de 4627 données pour la modélisation dynamique de ce nutriment (YAO et al., 2016b).

\subsubsection{Choix des meilleurs modèles}

Les résultats obtenus lors des simulations pour les deux scénarios étudiés sont résumés dans les tableaux 3 et 4 .

Tableau 1. Valeurs propres et statistiques associées de l'analyse en composantes principales (ACP) des six variables.

Table 1. Eigenvalues and related statistics from Principal Component Analysis (CPA) of the six variables.

\begin{tabular}{lcccc}
\hline Variable & Valeur propre & $\begin{array}{c}\text { Pourcentage } \\
\text { total (\%) }\end{array}$ & $\begin{array}{c}\text { Cumul des } \\
\text { valeurs propres }\end{array}$ & $\begin{array}{c}\text { Cumul } \\
(\%)\end{array}$ \\
\hline F1 & 2,048455 & 34,14092 & 2,048455 & 34,1409 \\
F2 & 1,324817 & 22,08029 & 3,373273 & 56,2212 \\
F3 & 1,042600 & 17,37666 & 4,415872 & 73,5979 \\
F4 & 0,953182 & 15,88637 & 5,369055 & 89,4842 \\
F5 & 0,629908 & 10,49846 & 5,998962 & 99,9827 \\
F6 & 0,001038 & 0,01730 & 6,000000 & 100,0000 \\
\hline
\end{tabular}

Tableau 2. Matrice de corrélation de Bravais-Pearson pour les six paramètres. Corrélations significatives marquées à $p<0,05$, $n=661$ (observations à VM ignorées).

Table 2. Bravais-Pearson correlation matrix for the six parameters. Significant correlations atp $<0,05, n=661$ (MV observations not taken into account).

\begin{tabular}{lcccccc}
\hline Paramètre & $\begin{array}{c}\text { Transparence } \\
(\mathbf{m})\end{array}$ & $\begin{array}{c}\mathbf{M E S}^{\mathbf{a}} \\
\left(\mathbf{m g} \cdot \mathbf{L}^{-1}\right)\end{array}$ & $\begin{array}{c}\text { Pluviométrie } \\
(\mathbf{m m})\end{array}$ & $\begin{array}{c}\text { Débit } \\
\text { Bandama }\end{array}$ & Chlorophylle $\boldsymbol{a}$ & $\begin{array}{c}\text { Phosphore } \\
\mathbf{m i n e ́ r a l ~}\left(\mathbf{m g} \cdot \mathbf{L}^{-1}\right)\end{array}$ \\
\hline Transparence & 1,00 & - & - & - & - & - \\
MES $\left(\mathrm{mg} \cdot \mathrm{L}^{-1}\right)$ & 0,13 & 1,00 & - & - & - & - \\
Pluviométrie $(\mathrm{mm})$ & 0,00 & 0,07 & 1,00 & - & - & - \\
Débit Bandama & $-0,02$ & $-0,11$ & 0,13 & 1,00 & - & - \\
Chlorophylle $a$ & 0,07 & 0,10 & 0,04 & 0,29 & 1,00 & - \\
Phosphore minéral $\left(\mathrm{mg} \cdot \mathrm{L}^{-1}\right)$ & 1,00 & 0,14 & $-0,00$ & $-0,01$ & 0,08 & 1,00 \\
\hline
\end{tabular}

${ }^{a}$ Matières en suspension 


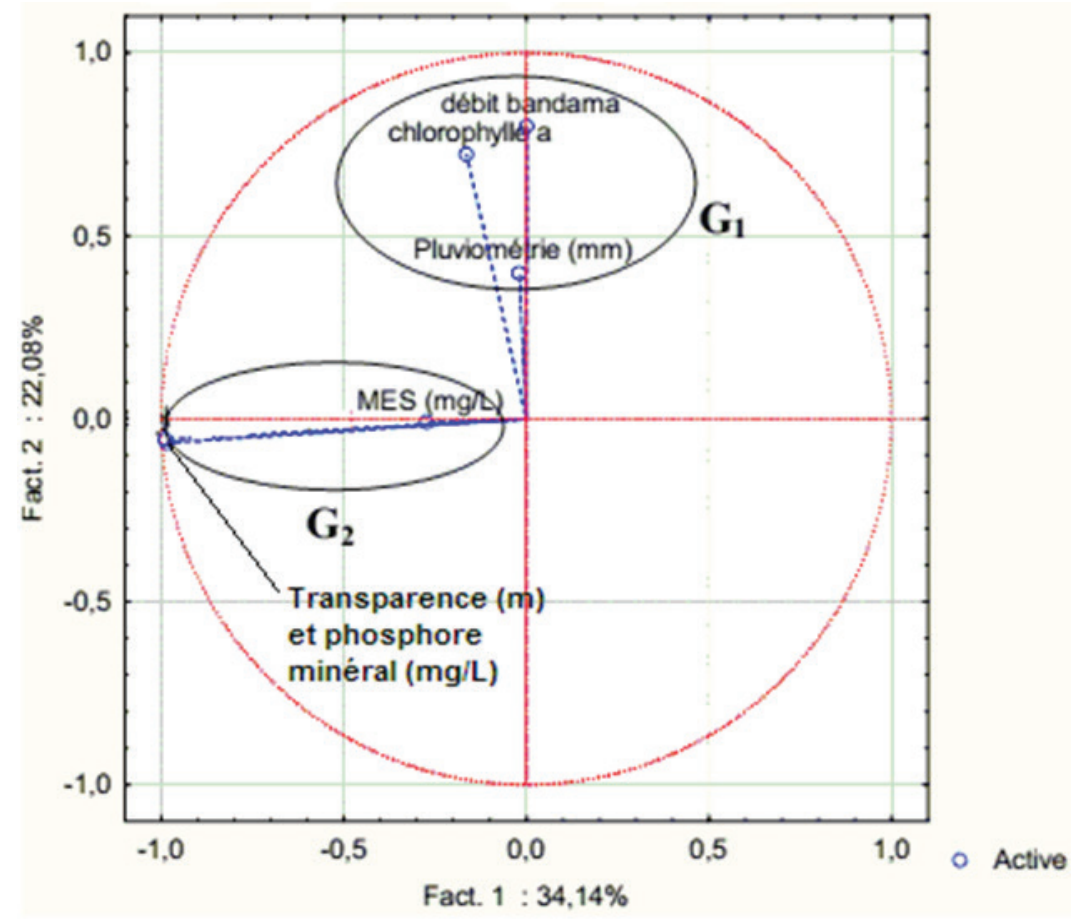

Figure 2. Projection des six paramètres dans le plan factoriel F1-F2.

Projection of the six parameters in the factorial plan F1-F2.

Tableau 3. Caractéristiques des différents modèles établis pour le scénario 1.

Table 3. Characteristics of different models for scenario 1.

\begin{tabular}{lcccc}
\hline Modèle & $\boldsymbol{R}_{\boldsymbol{a p p}}$ & $\boldsymbol{R}_{\text {val }}$ & $\boldsymbol{R}$ & $\mathbf{M C E}_{\mathrm{val}}$ \\
\hline $5-1-1$ & 0,7627733 & 0,666954 & 0,7148637 & 0,0527656 \\
$5-2-1$ & 0,8342692 & 0,7282708 & 0,78127 & 0,0481898 \\
$5-4-1$ & 0,8473805 & 0,7638996 & 0,80564 & 0,0388072 \\
$5-5-1$ & 0,8635018 & 0,77065881 & 0,8170803 & 0,0375235 \\
$5-5-1$ & 0,87649451 & 0,78388652 & 0,8301905 & 0,03543518 \\
$5-6-1$ & 0,89726342 & 0,82423793 & 0,8607507 & 0,03594269 \\
$5-7-1$ & 0,8962808 & 0,78988964 & 0,8430852 & 0,03555344 \\
$5-8-1$ & 0,9129483 & 0,8087992 & 0,8608737 & 0,0325818 \\
$5-9-1$ & 0,9119256 & 0,8424646 & 0,8771951 & 0,0336493 \\
$5-10-1$ & 0,9236231 & 0,8318741 & 0,8777486 & 0,0356175 \\
$5-11-1$ & 0,9134661 & 0,8303769 & 0,8719215 & 0,0357735 \\
$5-12-1$ & 0,9207153 & 0,8465921 & 0,8836537 & 0,0375071 \\
$5-13-1$ & 0,924897 & 0,8346651 & 0,8797811 & 0,03692 \\
5-14-1 & 0,9312592 & 0,8561259 & 0,8936925 & 0,034031 \\
5-15-1 & 0,9378751 & 0,8412397 & 0,8895574 & 0,0363974 \\
Min & - & - & - & 0,0325818 \\
Max & 0,9378751 & 0,8561259 & 0,8936925 & - \\
\hline
\end{tabular}


Tableau 4. Caractéristiques des différents modèles établis pour le scénario 2.

Table 4. Characteristics of different models for scenario 2.

\begin{tabular}{|c|c|c|c|c|c|c|c|c|}
\hline \multirow[t]{2}{*}{ Modèle } & \multicolumn{3}{|c|}{ Phosphore minéral $Y(t)$} & \multicolumn{3}{|c|}{ Phosphore minéral $Y(t+1)$} & \multicolumn{2}{|c|}{$\begin{array}{c}\text { Paramètres de validation } \\
\text { du modèle }\end{array}$} \\
\hline & $R_{a p p}(t)$ & $R_{v a l}(t)$ & $R_{1}^{a}$ & $R_{a p p}(t+1)$ & $R_{v a l}(t+1)$ & $\boldsymbol{R}_{2}^{\mathrm{b}}$ & $\boldsymbol{R}^{\prime \mathrm{c}}$ & $\mathrm{MCE}_{\mathrm{val}}$ \\
\hline $6-1-2$ & 0,116902 & 0,160424 & 0,138663 & 0,8019518 & 0,6817853 & 0,7418685 & 0,4402658 & 0,0708887 \\
\hline $6-2-2$ & 0,4566737 & 0,4563527 & 0,4565132 & 0,8344781 & 0,735948 & 0,785213 & 0,6208631 & 0,0513131 \\
\hline $6-3-2$ & 0,8807638 & 0,7764998 & 0,8286318 & 0,8036477 & 0,6823384 & 0,742993 & 0,7858124 & 0,0482674 \\
\hline $6-4-2$ & 0,8928117 & 0,7909248 & 0,8418682 & 0,8243294 & 0,7621466 & 0,793238 & 0,8175531 & 0,0465359 \\
\hline $6-5-2$ & 0,8780301 & 0,7778233 & 0,8279267 & 0,8682429 & 0,7669904 & 0,8176166 & 0,8227717 & 0,0453087 \\
\hline $6-6-2$ & 0,9147156 & 0,8288148 & 0,8717652 & 0,8833798 & 0,8219187 & 0,8526492 & 0,8622072 & 0,0452824 \\
\hline $6-7-2$ & 0,9259595 & 0,829384 & 0,8776717 & 0,8613111 & 0,7812874 & 0,8212993 & 0,8494855 & 0,0442414 \\
\hline $6-8-2$ & 0,91231 & 0,8079756 & 0,8601428 & 0,8875298 & 0,8159251 & 0,8517274 & 0,8559351 & 0,0440949 \\
\hline $6-9-2$ & 0,9353431 & 0,8407662 & 0,8880546 & 0,868482 & 0,7938278 & 0,8311549 & 0,8596048 & 0,0421144 \\
\hline $6-10-2$ & 0,9133696 & 0,8351317 & 0,8742506 & 0,9004123 & 0,8140193 & 0,8572158 & 0,8657332 & 0,0419518 \\
\hline $6-11-2$ & 0,9187918 & 0,8369972 & 0,8778945 & 0,8992213 & 0,8393925 & 0,8693069 & 0,8736007 & 0,0454758 \\
\hline $6-12-2$ & 0,9441109 & 0,8478214 & 0,8959662 & 0,8775604 & 0,8055298 & 0,8415451 & 0,8687556 & 0,0423015 \\
\hline $6-13-2$ & 0,9526877 & 0,8356778 & 0,8941828 & 0,8733399 & 0,7970497 & 0,8351948 & 0,8646888 & 0,0410594 \\
\hline $6-14-2$ & 0,9309743 & 0,8333354 & 0,8821549 & 0,9170915 & 0,8372391 & 0,8771653 & 0,8796601 & 0,0431562 \\
\hline $6-15-2$ & 0,9528209 & 0,8468072 & 0,899814 & 0,8914729 & 0,8266405 & 0,8590567 & 0,8794354 & 0,0411847 \\
\hline Max & 0,9528209 & 0,8478214 & 0,899814 & 0,9170915 & 0,8393925 & 0,8771653 & 0,8796601 & - \\
\hline Min & - & - & - & - & - & - & - & 0,0410594 \\
\hline
\end{tabular}

${ }^{a}$ Moyenne arithmétique de $R_{\text {app }}(t)$ et $R_{\text {val }}(t)$

${ }^{\mathrm{b}}$ Moyenne arithmétique de $R_{a p p}(t+1)$ et $R_{\text {val }}(t+1)$

c Moyenne arithmétique de $R_{1}$ et $R_{2}$

À l'analyse de ces résultats, l'on note que les valeurs les plus élevées de $R_{a p p}$ et $R_{v a l}$, de même que la valeur la plus faible de $\mathrm{MCE}_{\text {val }}$, sont obtenues pour des modèles distincts. Ainsi, les modèles 5-14-1 et 6-14-2 représentent-ils les meilleurs respectivement pour les scénarios 1 et 2 . Les représentations architecturales de ces deux modèles sont données par la figure 3 .

\subsubsection{Validation finale des modèles}

En se référant au tableau 5, les modèles 5-14-1 et 6-14-2 respectent tous les critères de validation de modèles énoncés dans la section 2.2.2.2. Ainsi, sont-ils tous deux validés.

Pour le scénario 1, le modèle 5-14-1 explique à 93,12 \% les variations spatiotemporelles des cinq paramètres d'entrée mise en jeu et prédit à 73,30\% l'évolution statique du phosphore minéral dans la baie lagunaire de Tiagba. Quant au modèle 6-14-2 destiné au scénario 2, il explique à 93,10\% et 91,71\% la variation spatiotemporelle des six paramètres d'entrée mise en jeu respectivement pour le mois $(t)$ et le mois suivant $(t+1)$. Il prédit à $69,44 \%$ et 70,10\% la dynamique du phosphore minéral au mois $(t)$ et au mois $(t+1)$ respectivement. Le coefficient de corrélation entre les valeurs expérimentales et celles calculées par le modèle est de 0,80545 pour le modèle 5-14-1 et 0,85761 pour 6-14-2 (Figure 4).

\subsection{Discussion}

Les modèles 5-14-1 et 6-14-2 permettent de prédire respectivement les évolutions statique et dynamique du phosphore dans les eaux de la baie lagunaire de Tiagba avec des marges d'erreurs comprises entre $26,67 \%$ et $30 \%$. Ces marges d'erreurs pourraient s'expliquer par la sous-estimation de certains processus liés au cycle biogéochimique du phosphore dans cette étude tel que le flux de transfert du phosphore dans les systèmes sol-sédiment et sédiment-eau, les activités benthiques, la mobilité du phosphore dans les sédiments, etc.

Ces résultats sont jugés relativement satisfaisants dans la mesure où ils permettent de confirmer la grande influence des apports météorites (environ $70 \%$ ) sur la dynamique du phosphore minéral dans les eaux de cette baie lagunaire, déjà soupçonné par YAO (2011). Ainsi, ces différents modèles établis peuvent-ils servir dans le cadre d'une prise de décisions visant à la réhabilitation, la protection et la gestion à court et long terme de cette baie lagunaire. L'une des techniques susceptibles d'être proposée dans ce cadre est d'y favoriser l'apport du fleuve Bandama en élargissant et draguant régulièrement le canal d'Assagny. En effet, YAO (2011) a noté que cet apport fluviatile inhiberait la croissance de ce nutriment, en même temps que celle du phytoplancton. Aussi, faudra-t-il limiter voire interdire l'utilisation des fertilisants phosphorés sur le bassin versant de cette baie lagunaire, où la pratique agricole est intense. 
a

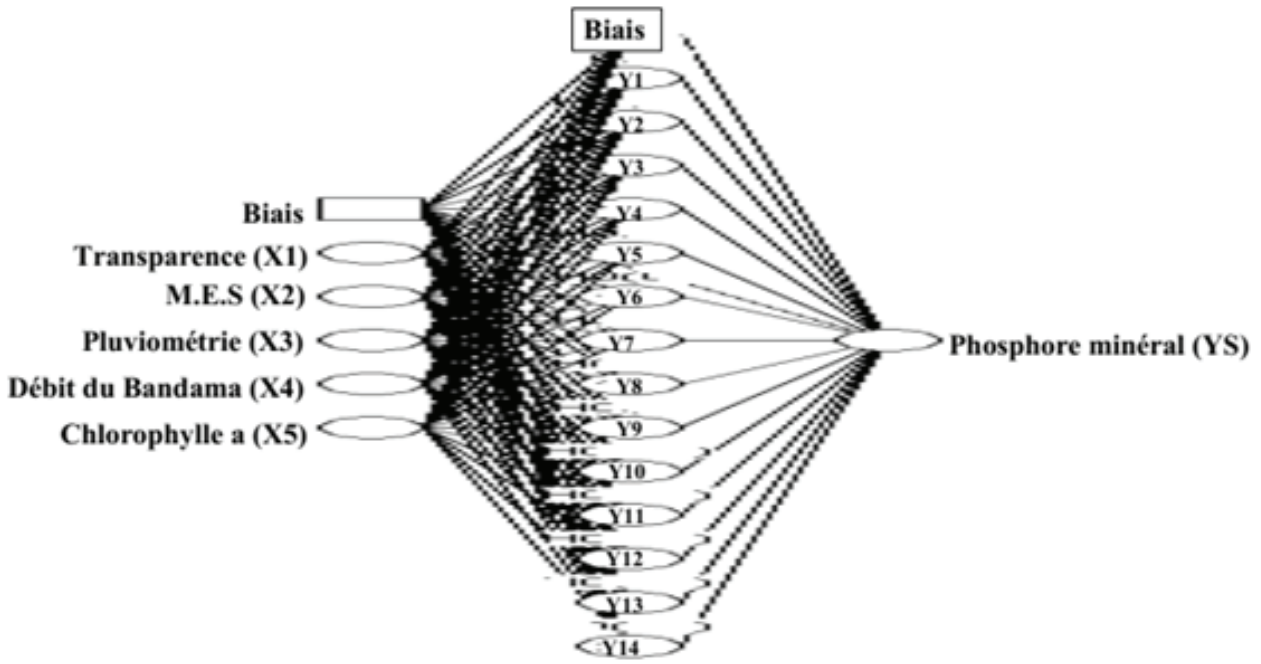

b

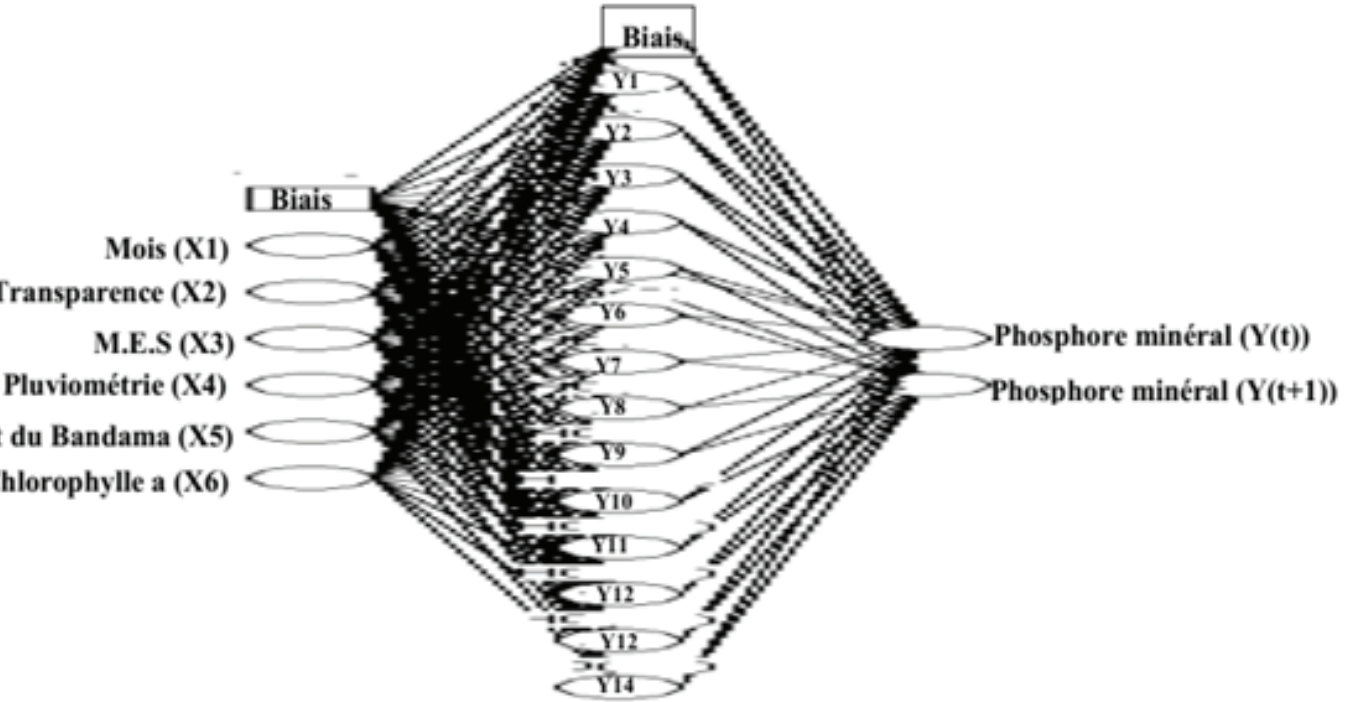

Figure 3. Architecture des modèles a) 5-14-1 et b) 6-14-2. Architecture of models a) 5-14-1 and b) 6-14-2.

Tableau 5. Validation finale des modèles 5-14-1 et 6-14-2.

Table 5. Final validation of models 5-14-1 and 6-14-2.

\begin{tabular}{lclll}
\hline \multirow{2}{*}{$\begin{array}{l}\text { Paramètre de validation du } \\
\text { modèle }\end{array}$} & \begin{tabular}{l} 
Modèle \\
\cline { 2 - 5 }
\end{tabular} & $\begin{array}{l}\text { Obsernario 1 } \\
\text { rapport aux critères } \\
\text { de validation }\end{array}$ & $\begin{array}{l}\text { Modèle } \\
\mathbf{6 - 1 4 - 2}\end{array}$ & $\begin{array}{l}\text { Observation par } \\
\text { rapport aux critères } \\
\text { de validation }\end{array}$ \\
\hline $\begin{array}{l}R^{2} \text { en phase de validation à } t+1 \\
\left(R_{\text {val }}^{2}\right)\end{array}$ & 0,7330 & $>0,5$ & 0,6944 mois $(t)$ & $>0,5$ \\
$\begin{array}{l}\text { Moyenne carrée des erreurs en phase } \\
\text { test }(\text { MCE test })\end{array}$ & 0,0392 & Très faible & 0,7010 mois $(t+1)$ & Très faible \\
$\begin{array}{l}\text { Racine des MCE en phase test } \\
\text { (RMCE test) }\end{array}$ & 0,1993 & Très faible & 0,2026 & Très faible \\
$\begin{array}{l}\text { Erreur moyenne de prévision } \\
\left(e_{m o y}\right)\end{array}$ & 0,0656 & Très faible & 0,0652 mois $(t)$ & Très faible \\
$\begin{array}{l}\text { Erreur moyenne de déviation }(\Delta) \\
(\%)\end{array}$ & 2,5564 & $<10$ & 0,0925 mois $(t+1)$ & \\
$\begin{array}{l}\text { État du modèle } \\
\text { (validé ou non validé) }\end{array}$ & Validé & - & 2,6920 mois $(t)$ & $<10$ \\
\hline
\end{tabular}



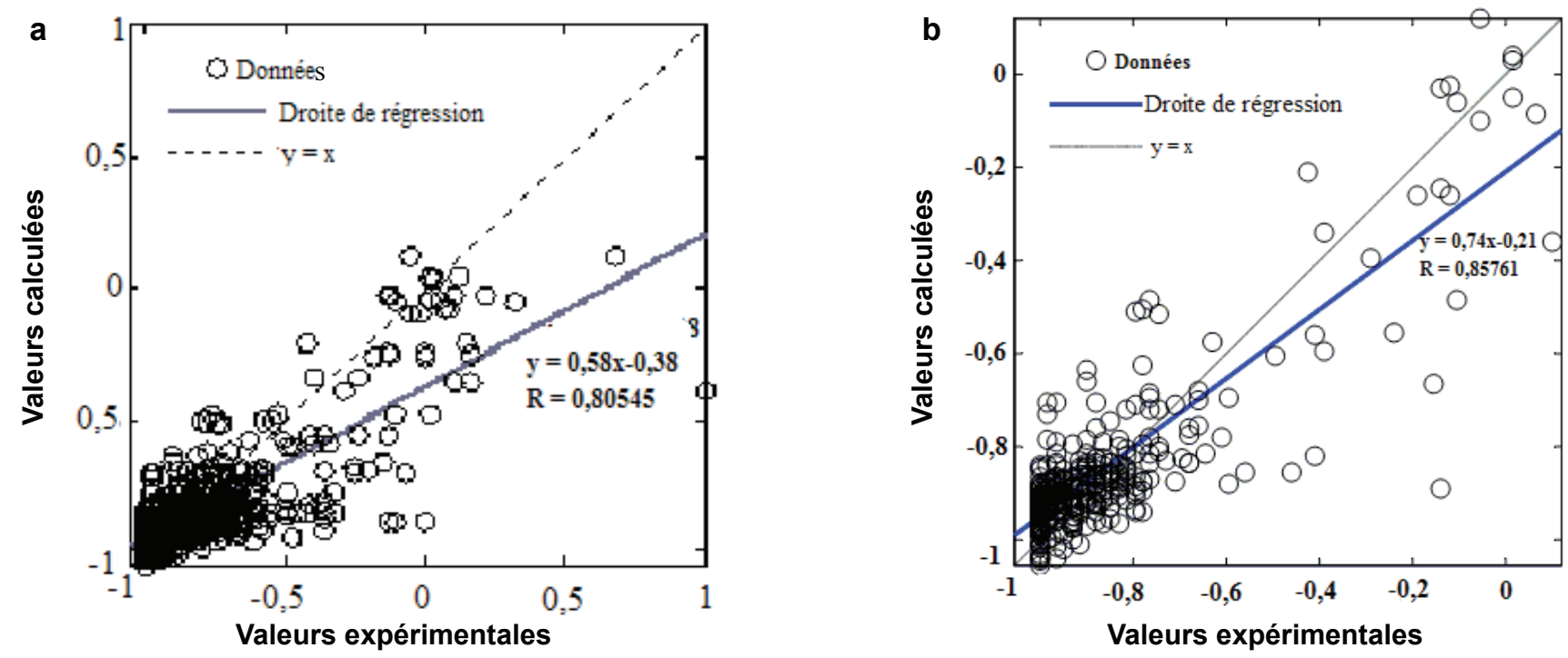

Figure 4. Représentation des valeurs expérimentales normalisées en fonction des valeurs calculées normalisées pour les modèles : a) 5-14-1 et b) 6-14-2.

Representation of normalized experimental values as function of normalized predicted values for models: a) 5-14-1 and b) 6-14-2.

Par ailleurs, l'utilisation des RNA dans cette étude est d'une originalité dans la mesure où la quasi-majorité des modèles consacrés à la modélisation du cycle biogéochimique du phosphore dans les eaux de surface sont des modèles de connaissances (ROBSON, 2014). Également, contrairement à ceux-ci (GROWA/MEPhos [GUSE et al., 2007; TETZLAFF, 2006], MONERIS [BEHRENDT et al., 2000], CASA model [WANG et al., 2010], SWAT model [FONTAINE et al., 2002; FREIHOEFER et McGINLEY, 2009; SANTHI et al., 2001], WEE et WEEE [DUCHESNE et al., 2016], etc.) qui ont un champ d'action réduit aux plans d'eau pour lesquels ils ont été établis, les RNA sont dans leur quintessence applicables pour la modélisation des processus aquatiques de tous genres dans n'importe quel plan d'eau. Ainsi, sont-ils de véritables outils importants pour la gestion des bassins des eaux de surfaces.

\section{CONCLUSION}

Cette étude vient encore une fois démontrer la capacité des RNA à modéliser les phénomènes aussi complexes que les processus biogéochimiques tel que celui du phosphore minéral. Les deux modèles établis, jugés satisfaisants, peuvent être utilisés en complémentarité avec ceux de YAO et al. (2016a) dans le cadre de la lutte contre l'eutrophisation d'une part, et servir à la gestion de ce plan d'eau dans le cadre général de son développement durable d'autre part.

\section{RÉFÉRENCES BIBLIOGRAPHIQUES}

AICHOURI I., A. HANI, N. BOUGHERIRA, L. DJABRI, H. CHAFFAI et S. LALLAHEM (2015). River flow model using artificial neural networks. Energy Procedia, 74, 1007 1014.

ABEL J.M., D. ÖZKUNDACI et D.P. HAMILTON (2010). Nitrogen and phosphorus limitation of phytoplankton growth in New Zealand lakes: Implications for eutrophication control. Ecosyst., 13 (7), 966-977.

BASHEER I.A. et M. HAJMEER (2000). Artificial neural networks: Fundamentals, computing, design, and application. J. Microbiol. Methods, 43, 3-31.

BEHRENDT H., P. HUBER, M. KORMMILCH, D. OPITZ, O. SCHMOLL, G. SCHOLZ et R. UEBE (2000). Nutrient emissions into river basins of Germany. Environmental Research of the Federal Ministry of the Environment, Nature Conservation and Nuclear Safety, Institute of Freshwater Ecology and Inland Fisheries, Texte 23/00, Research Report 29625 515, UBA-FB 99-087/e, Berlin, Allemagne, 13 p.

CABRITA M.T., A. SILVA., P.B. OLIVEIRA, M.M. ANGÉLICO et M. NOGUEIRA (2015). Assessing eutrophication in the Portuguese Continental Exclusive Economic Zone within the European Marine Strategy Framework Directive. Ecol. Indic., 58, 286-299. 
CAO X., Y. WANG, J. HE, X. LUO et Z. ZHENG (2016). Phosphorus mobility among sediments, water and cyanobacteria enhanced by cyanobacteria blooms in eutrophic Lake Dianchi. Environ. Pollut., 219, 580-587.

CHANG N.B., G. MOHIUDDIN, A.J. CRAWFORD, K. BAI et K.R. JIN (2015). Diagnosis of the artificial intelligencebased predictions of flow regime in a constructed wetland for stormwater pollution control. Ecol. Inform., 28, 42-60.

COBLE A.A., A.M. MARCARELLI et E.S. KANE (2015). Ammonium and glucose amendments stimulate dissolved organic matter mineralization in a Lake Superior tributary. J. Great Lakes Res., 41 (3), 801-807.

CARMOUZE J.P. et J.P. CAUMETTE (1985). Les effets de la pollution organique sur les biomasses et activités du phytoplancton et des bactéries hétérotrophes dans la lagune Ébrié (Côte d'Ivoire). Rev. Hydrobiol. Trop., 18 (3), 183-211.

DUCHESNE S., B. TOUMBOU et J.P. VILLENEUVE (2016). Validation and comparison of different statistical models for the prediction of water main pipe breaks in a municipal network in Québec, Canada. Rev. Sci. Eau, 29 (1), 1-89.

FONTAINE T.A., T.S. CRUICKSHANK, J.G. ARNOLD et R.H. HOTHCHKISS (2002). Development of a snowfall/ snowmelt routine for mountainous terrain for the soil water assessment tool (SWAT). J. Hydrol., 262, 209-223.

FOTIOU T., T.M. TRIANTIS, T. KALOUDIS et A. HISKIA (2015). Evaluation of the photocatalytic activity of $\mathrm{TiO}_{2}$ based catalysts for the degradation and mineralization of cyanobacterial toxins and water off-odor compounds under UV-A, solar and visible light. Chem. Eng. J., 261, 17-26.

FREIHOEFER A. et P. McGINLEY (2009). Phosphorus loading model for Lake Eau Claire and Lake Altoona. Center for Watershed Science and Education, University of Wisconsin - Stevens Point, Wisconsin, États-Unis, 99 p.

GUSE B., A. BRONSTERT, M. RODE, B. TETZLAFF et F. WENDLAND (2007). Application of two phosphorus models with different complexities in a mesoscale river catchment. Adv. Geosci., 11, 77-84.

GOODY D.C., D.J. LAPWORTH, S.A. BENNETT, T.H.E. HEATON, P.J. WILLIAMS et B.W.J. SURRIDGE (2016). A multi-stable isotope framework to understand eutrophication in aquatic ecosystems. Water Res., 88, 623633.
HUANG J. et J. GAO (2017). An ensemble simulation approach for artificial neural network: An example from chlorophyll a simulation in Lake Poyang, China. Ecol. Inform., 37, 52-58.

HUO S., Z. HE, J. SU, B. XI et C. ZHU (2013). Using artificial neural network models for eutrophication prediction. Procedia Environ. Sci., 18, 310-316.

KASPERSEN B.S., T.B. CHRISTENSEN, A.M. FREDENSLUND, H.B. MøLLER, M.B. BUTTS, N.H. JENSEN et T. KJAER (2016). Linking climate change mitigation and coastal eutrophication management through biogas technology: Evidence from a new Danish bioenergy concept. Sci. Total. Environ., 541, 124-131.

LEVENBERG K. (1944). A method for the solution of certain problems in least squares. Quart. Appl. Math., 2, 164-168.

LIU C., S. SHAO, Q. SHEN, C. FAN, L. ZHANG et Q. ZHOU (2016a). Effects of riverine suspended particulate matter on the post-dredging increase in internal phosphorus loading across the sediment-water interface. Environ. Pollut., 211, 165-172.

LIU C., J. ZHONG, J. WANG, L. ZHANG et C. FAN (2016b). Fifteen-year study of environmental dredging effect on variation of nitrogen and phosphorus exchange across the sediment-water interface of an urban lake. Environ. Pollut., 219, 639-648.

MA Z., X. SONG, R. WAN, L. GAO et D. JIANG (2014). Artificial neural network modeling of the water quality in intensive Litopenaeus vannamei shrimp tanks. Aquaculture, 433, 307-312.

MANDAL S., S.S. MAHAPATRA et R.K. PATEL (2015). Enhanced removal of $\mathrm{Cr}(\mathrm{VI})$ by cerium oxide polyaniline composite: Optimization and modeling approach using response surface methodology and artificial neural networks. J. Environ. Chem. Eng., 3 (2), 870-885.

MANSSOURI T., H. SAHBI, I. MANSSOURI et B. BOUDAD (2015). Utilisation d'un modèle hybride basé sur la RLMS et les RNA-PMC pour la prédiction des paramètres indicateurs de la qualité des eaux souterraines, cas de la nappe de Souss-Massa-Maroc. Eur. Sci. J., 11, 3546.

MARQUARDT D. (1963). An algorithm for least-squares estimation of nonlinear parameters. J. Soc. Indust. Appl. Math., 11, 431-441. 
MELLANDER P.E., P. JORDAN, M. SHORE, N.T. McDONALD, D.P. WALL, G. SHORTLE et K. DALY (2016). Identifying contrasting influences and surface water signals for specific groundwater phosphorus vulnerability. Sci. Total. Environ., 541, 292-302.

MULLER A.C. et D.L. MULLER (2015). Forecasting future estuarine hypoxia using a wavelet based neural network model. Ocean Modell., 96 (2), 314-323.

NOUMI M. (2004). Painlevé equations through symetry. Translations of Mathematical Monographs. Vol. 223, American Mathematical Society (AMS), États-Unis, 156 p.

PINEDO S., R. ARÉVALO et E. BALLESTEROS (2015). Seasonal dynamic of upper sublittoral assemblages on Mediterranean rocky shores along a eutrophication gradient. Estuar. Coast. Shelf Sci., 161, 93-101.

PIZARRO J., P.M. VERGARA, S. CERDA et D. BRIONES (2016). Cooling and eutrophication of southern Chilean lakes. Sci. Total. Environ., 541, 683-691.

ROBSON J.B. (2014). State of the art in modelling of phosphorus aquatic systems: Review, criticisms and commentary. Environ. Model. Softw., 61, 339-359.

ROLLAND D.C., J. HAURY, P. MARMONIER et Y. LAGADEUC (2015). Effect of macrophytes on flow conditions and deposition of suspended particles in small streams: An experimental study using artificial vegetation. Rev. Sci. Eau, 28 (3), 231-245.

SANTHI C., J.G. ARNOLD, J.R. WILLIAMS, W.A. DUGAS, R. SRINIVASAN et L.M. HAUCK (2001). Validation of the SWAT model on a large river basin with point and nonpoint sources. J. Am. Water Resour. Assoc., 37 (5), 1169 1188.

SHOULIANG H., H. ZHUOSHI, S. JING, X. BEIDOU et Z. CHAOWEI (2013). Using artificial neural network models for eutrophication prediction. Procedia Environ. Sci., 18, 310-316.

SONG Y., Y. DENG et C. JUNG (2016). Mitigation and degradation of natural organic matters (NOMs) during ferrate(VI) application for drinking water treatment. Chemosphere, 146, 145-153.

SUNOHARA M.D., N. GOTTSCHALL, E. CRAIOVAN, G. WILKES, E. TOPP, S.K. FREY et D.R. LAPEN (2016). Controlling tile drainage during the growing season in Eastern Canada to reduce nitrogen, phosphorus, and bacteria loading to surface water. Agric. Water Manag., 178, 159-170.
SUTELA T., T. VEHANEN et M. RASK (2016). A littoral fish index that responds to eutrophication in boreal lakes. Fisheries Res., 173 (1), 88-92.

TETZLAFF B. (2006). Die Phosphatbelastung großer Flusseinzugsgebiete aus diffusen und punktuellen Quellen. Schriften des Forschungszentrums Juelich, Reihe Umwelt/ Environment, Juliers, Allemagne, Vol. 65, 287 p.

ULRICH A.E., D.F. MALLEY et P.D. WATTS (2016). Lake Winnipeg basin: Advocacy, challenges and progress for sustainable phosphorus and eutrophication control. Sci. Total. Environ., 542 (B), 1030-1039.

WANG L. et T. LIANG (2016). Distribution patterns and dynamics of phosphorus forms in the overlying water and sediment of Dongting Lake. J. Great Lakes Res., 42 (3), 565-570.

WANG Y.P., R.M. LAW et B. PARK (2010). A global model of carbon, nitrogen and phosphorus cycles for the terrestrial biosphere. Biogeosciences, 7, 2261-2282.

XU Y., C. MA, Q. LIU, B. XI, G. QIAN, D. ZHANG et S. HUO (2015). Method to predict key factors affecting lake eutrophication - A new approach based on Support Vector Regression model. Int. Biodeterior. Biodegradation, 102, 308-315.

YAO M.K. (2011). Évaluation et modélisation de l'eutrophisation dans une baie lagunaire tropicale apparentée lacustre : cas de la baie lagunaire de Tiagba (Côte d'Ivoire). Thèse de doctorat, Univ. Félix Houphouët-Boigny, Côte d'Ivoire, 242 p.

YAO M.K., K.L. AKPETOU, Y.S. BROU, D.C AKMEL, A. TROKOUREY et K.B. YAO (2016a). Eutrophication modeling by new approach in tropical lagoon bay: Case of Tiagba Lagoon Bay (Ebrie Systeme, Côte d'Ivoire). Aust. J. Basic Appl. Sci., 10 (13), 37-44.

YAO Y., P. WANG, C. WANG, J. HOU, L. MIAO, Y. YUAN, T. WANG et C. LI (2016b). Assessment of mobilization of labile phosphorus and iron across sediment-water interface in a shallow lake (Hongze) based on in situ high-resolution measurement. Environ. Pollut., 219, 873-882.

ZUO J., J. SONG, H. YUAN, X. LI, N. LI et L. DUAN (2016). Particulate nitrogen and phosphorus in the East China Sea and its adjacent Kuroshio waters and evaluation of budgets for the East China Sea Shelf. Cont. Shelf Res., $131,1-11$.

ZHANG G.P. (2003). Time series forecasting using a hybrid ARIMA and neural networkmodel. Neurocomputing, 50, 159-175. 